\title{
Differentiation of conductive cells: a matter of life and death
}

\author{
Jung-ok Heo ${ }^{1,2}$, Bernhard Blob ${ }^{1}$, Yka Helariutta ${ }^{1,2}$
}

${ }^{1}$ Sainsbury Laboratory, Cambridge University, Bateman Street, Cambridge CB2 1LR, UK

${ }^{2}$ Institute of Biotechnology, University of Helsinki, 00014 Helsinki, Finland

Corresponding author: Yka Helariutta (yrjo.helariutta@slcu.cam.ac.uk)

Co-first authors: Jung-ok Heo (jungok.heo@slcu.cam.ac.uk) and Bernhard Blob (bernhard.blob@slcu.cam.ac.uk) 


\begin{abstract}
Two major conducting tissues in plants, phloem and xylem, are composed of highly specialized cell types adapted to long distance transport. Sieve elements (SEs) in the phloem display a thick cell wall, callose-rich sieve plates and low cytoplasmic density. SE differentiation is driven by selective autolysis combined with enucleation, after which the plasma membrane and some organelles are retained. By contrast, differentiation of xylem tracheary elements (TEs) involves complete clearance of the cellular components by programmed cell death followed by autolysis of the protoplast; this is accompanied by extensive deposition of lignin and cellulose in the cell wall. Emerging molecular data on TE and SE differentiation indicate a central role for NAC and MYB type transcription factors in both processes.
\end{abstract}




\section{Introduction}

Plants have evolved highly specialized vascular tissues which enable the translocation of various molecules that are vital for their growth and survival. Phloem, one of principal components of the vascular system, transports sucrose from photosynthetic organs to actively developing ones, such as flowers, fruits, buds, and roots. Another major conduit is the xylem, a path through which water absorbed by the root is transported to the above-ground tissues. Recent studies have shed light on the role of phloem and xylem as conduits mediating long-distance communication. Various plant growth substances, such as phytohormones, small non-coding RNAs, proteins, and peptides were shown to be transported through these conduits [1-5]. Both conduits undergo specialized differentiation by which internal flow resistance is minimized and the cell walls become bolstered, enabling efficient molecular trafficking through the conduits. During the differentiation of phloem sieve elements (SEs), some organelles are selectively degraded and others are retained in a modified form. This differentiation process allows phloem to transport molecules through their symplastic space. By contrast, xylem tracheary elements (TEs) undergo complete clearance of their cellular components during differentiation, leaving only an apoplastic transport route available. In addition, the thick and lignified cell walls of xylem vessels and fibres provide mechanical support for the plant.

Recent experiments have lent support to pressure flow theory, a long-standing hypothesis which explains the transport mechanism in the phloem [6]. According to the theory, bulk flow in SEs is driven by a difference in osmotic pressure, which is high in source tissues, where sucrose is loaded, and low in sink tissues, where sucrose is unloaded. Cohesion-tension theory offers an explanation for the upward translocation of water through the xylem, proposing that the evaporation of water at the leaf surface due to transpiration creates a tension (negative hydrostatic pressure) which drives the ascent of sap. A stream of water is maintained in xylem TEs by the cohesive and adhesive properties of water [7]. In the following sections, we will describe the ontogeny of phloem and xylem tissues and discuss the molecular mechanisms regulating the differentiation of each conduit. 


\section{Specification and maintenance of the vascular tissue pattern}

In Arabidopsis, vascular cell fate is determined very early in embryogenesis, at the globular stage. Subsequent periclinal divisions give rise to xylem, phloem, and procambium precursors in a spatially organized manner. The spatial coordination by oriented cell divisions is attributed to the interplay between two phytohormones, cytokinins and auxin [8], which are also crucial to maintain a stable vascular pattern in the post-embryonic root by establishing two distinct hormone signalling domains

[9-11]. The diverse patterns of vascular tissue organization in different species have been reviewed in $[12,13]$. 


\section{Differentiation of phloem and xylem - cytological changes}

In contrast to xylem TEs, in which programmed cell death (PCD) followed by autolysis results in the total loss of protoplasmic contents, sieve tube elements undergo selective autolysis, retaining a few organelles and the plasma membrane (Figure 1). Before the onset of the differentiation, sieve element precursor cells do not show any obvious cytological differences compared with neighbouring cells. As the sieve tubes begin differentiating, the stroma of their plastids become electron-transparent, making the plastids discernible [14] (Figure 1a). Sieve element plastids are divided into two types: S-type plastids, which contain starch inclusions, and P-type plastids, which contain proteinaceous inclusion bodies and are typical of monocots [15]. Another feature noticeable early in SE differentiation is the presence of phloem protein (P-protein) bodies, agglomerates of protein tubules or filaments. In mature SEs, P-proteins are found in various forms, such as tubules, filaments, and amorphous or crystalline structures [16]. In most angiosperms, P-protein bodies disperse into filaments or a bundle of filaments and eventually align with the plasma membrane at the cell's side walls or on the sieve plates [17] (Figure 1a).

SE differentiation culminates in nuclear degradation. In protophloem SEs in the Arabidopsis primary root, enucleation is marked by the deformation of the nucleus. Prior to degradation, the nuclear envelope becomes disorganized and the nucleus shrinks. During enucleation, the nuclear contents diffuse into the cytoplasm, where they are degraded, leaving behind a remnant of the nuclear envelope (Figure 1a). The entire process takes 10 minutes [18]. When the nucleus changes its shape, the nucleolus becomes fragmented. The mitochondria also gradually change shape during nuclear degradation [18], and the ER undergoes structural changes by making stacks, losing ribosomes and migrating toward the wall [14] (Figure 1a). The appearance of perinuclear complexes composed of vacuoles and many other organelles was shown to coincide with nuclear disintegration (Figure 1a). No signs of a large central vacuole were observed, but small lytic vacuoles were shown to persist before and after enucleation, suggesting that the mechanism is slightly different from PCD [18]. In mature SEs, modified organelles such as the plastids and mitochondria are eventually aligned with the parietal walls of the cell (Figure 1a).

In angiosperms, SEs are functionally associated with specialized parenchyma cells named companion cells (CCs). Unlike SEs, which lose many of their organelles, CCs are characterized by their increased cellular density. A large nucleus and an increased number of ribosomes, mitochondria, ER, and plastids are observed in CCs [19] (Figure 1a). It has long been proposed that CCs support SEs to keep them viable. However, details about how they are functionally related remain far from being understood.

A detailed picture of the cytological changes during TE differentiation have been pieced together by studying an in vitro cell culture system of Zinnia elegans. The start of TE differentiation is marked by a swelling of the vacuole, which leads to altered tonoplast permeability and eventually vacuole collapse. The release of hydrolytic enzymes into the cytoplasm increases its acidity, activating cytoplasmic enzymes, which results in dilation of the ER and Golgi and the complete degeneration of other organelles [20,21] (Figure 1b). Cytoplasmic streaming is no longer active after the vacuole collapses. It has been proposed that the degradation of nucleus in TE differentiation is related to a post-mortem autolysis event rather than being a controlled degradation [22] (Figure 1b). 


\section{Differentiation of phloem and xylem - cell wall elaboration}

The initiation of differentiation in both SEs and TEs is delineated by the thickening of their walls. Although there is considerable variation in the walls' structure and composition, the walls of mature SEs are thought to consist of primary cell wall components such as cellulose and pectin. However, some species were shown to have lignified thick cell walls in SEs [14]. The ends of SE cells undergo significant modification in the formation of sieve plates, when the plasmodesmata (PD) at the junctions of SEs become enlarged and perforated. These perforated pores begin to be visible once the nucleus has degraded (Figure 1a). Callose (beta-1,3-glucan), a constituent of cell walls, is deposited on sieve plates as well as at PD on the lateral cell wall [23].

Lateral sieve areas in mature sieve tube elements provide a symplastic path to CCs through branched PD. These modified PD, also known as phloem pore units (PPU), link multiple pores from the side of companion cell to a single channel from the sieve tube element (Figure 1a). PPUs allow short-range cell-to-cell communication. In fact, a wide range of molecules, such as small RNAs and even exogenous protein such as GFP have been shown to be transported from CCs to SEs through PPUs. The size exclusion limit (SEL) of these modified PD is slightly larger than other PD, allowing proteins as large as $67 \mathrm{kDa}$ to move through them [4,24]. Some RNA molecules have been shown to need RNA-binding proteins for symplastic transport, suggesting that molecular trafficking between SEs and CCs is selective [4].

Unlike phloem SEs, which exhibit thick walls consisting mainly of primary cell wall material, the diverse xylem elements are distinguished by unique patterns of secondary cell wall (SCW) deposition [25]. In addition, while tracheids, an evolutionarily ancient form of TEs, are connected only by pitpairs on their common walls, vessel elements are connected by perforation plates at their junctions [26]. The secondary walls of vessels and fibres are composed of cellulose, hemicelluloses and phenolic lignin polymers deposited in special patterns which can be used to clearly distinguish the vessel types [27,28]. SCW patterning depends on the orientation of cellulose microfibrils, which is directed by the arrangement of microtubules [29,30] (Figure 1b). Following cellular autolysis, the primary cell walls are partially hydrolysed, especially at positions free of secondary cell wall [22]. Details about secondary cell wall formation and fibre differentiation are well described in [22,31]. 


\section{Molecular regulation of phloem and xylem differentiation}

Despite general similarities in SE and TE differentiation, such as cell wall thickening, autolysis and the loss of the nucleus, most of regulatory components vary.

The MYB transcription factor (TF) ALTERED PHLOEM DEVELOPMENT (APL) is a key regulator and promoter of SE differentiation [32]. The loss-of-function mutant has cells with mixed SE and TE identity in the position of SEs and is seedling lethal [32,33]. Two members of the plant-specific NAC TF family, NAC45 and NAC86, are downstream targets of APL and are expressed in differentiating SEs and in the phloem pole pericycle cells. They redundantly regulate the expression of NACDEPENDENT EXONUCLEASE 1, 2 and 4 (NENs) and control the translocation of NEN2 from the cytosol to the nucleus. The NAC-NEN pathway was shown to regulate nuclear degradation during SE differentiation (Figure 2a). Similar to the apl mutant, a double knockout mutant, nac45-2 nac86, exhibited seedling lethality and retained nuclei in SEs, while mitochondrial shape changes and the formation of sieve plates occur normally [18]. Proper transformation of PD into SPPs connecting the SEs is affected by the level of choline, which depends on the expression of CHOLINE TRANSPORTER LIKE 1 (CHER1) [34]. However, the detailed cellular processes influenced by CHER1 and the choline level have yet to be revealed. In addition, callose deposition by CALLOSE SYNTHASE 7 (CALS7) is required for correct formation of SPPs [35].

The recent development of a culture system with inducible ectopic phloem differentiation has facilitated the identification of new regulatory elements. In this system, NAC020 was identified as an early, SE-specific NAC TF that potentially acts as a negative regulator of APL [36] (Figure 2a). Furthermore, several genes have recently been found to regulate early SE development, including BREVIX RADIX (BRX), OCTOPUS (OPS), CLAVATA3/ENDOSPERM SURROUNDING REGION 45 (CLE45) and its receptor BARELY ANY MERISTEM3 (BAM3). So far, the evidence implicates BRX as an upstream regulator of CLE45/BAM3 signalling, while MEMBRANE-ASSOCIATED KINASE REGULATOR 5 was recently shown to be a downstream amplifier of the CLE45 signal [37]. In the Arabidopsis root, BRX and OPS are plasma-membrane-associated proteins which are polarly localized rootward and shootward, respectively, but their regulatory interaction is not yet fully understood [38,39]. BRX is thought to translate auxin signalling in a contextual manner, while OPS is a positive regulator of SE differentiation and might activate BRI1-EMS-SUPPRESSOR 1 (BES1) by sequestering its repressor BRINSENSITIVE 2 [38,40,41]. The small mobile peptide CLE45 suppresses SE differentiation in SE precursor cells [42] (Figure 2a). Loss-of-function or overexpression of these genes results in differentiated SE strands containing undifferentiated cells which interrupt the continuity of the cell file [39-43]. In addition, COTELYDON VASCULAR PATTERN2 (CVP2) acts genetically upstream of OPS by controlling the level of phosphatidylinositol-4,5biphosphate ( $\mathrm{PIP}_{2}$ ), although the exact mechanism of this control is not yet understood (Figure 2a). A double mutant of CVP2 and its homolog CVP2-like1 (CVL1) has discontinuous SE cell files, similar to the ops and brx mutants [44].

The role of auxin response in TE fate determination and the initiation of differentiation, and the tight control both spatially and temporally are well described [45-50]. CLE41 and CLE44, small peptides which contain the TRACHEARY ELEMENT DIFFERENTIATION INHIBITORY FACTOR sequence in their C-terminus, were shown to suppress TE differentiation in Arabidopsis, similar to the effect of CLE45 on SE differentiation [51]. Interestingly, this signalling involves the suppression of $B E S 1$, which is required for the differentiation not only of SEs but also of TEs from procambial and cambial cells [52]. TE differentiation is controlled by two other members of the NAC TF family, 
VASCULAR-RELATED NAC-DOMAIN6/7 (VND6/7), which were both shown to be sufficient to induce differentiation of TEs in other cell types. $V N D 6 / 7$ control the expression of genes responsible for secondary cell wall thickening and PCD, resulting in temporal co-occurrence of these two events [53-55]. The MYB TFs MYB46 and MYB83 act downstream of VND6/7 to regulate secondary cell wall synthesis [56,57]. Another NAC TF, VND-INTERACTING2 interacts with VND7 and represses TE differentiation [58]. Furthermore, the level of the polyamine thermospermine, which is controlled by the essential biosynthetic enzyme ACAULIS5 (ACL5), regulates the patterning of SCW and delays cell death [59]. Thermospermine and ACL5 are thought to act through SUPRESSOR OF ACAULIS51 (SAC51), which is also a direct target of VND7 [60] (Figure 2b). Xylogen, a secreted proteoglycan first isolated from Zinnia cultures and active in both monocots and dicots, is another important signal in TE differentiation. It is polarly localized towards the shoot apex on the plasma membrane, and only the homolog ARABIDOPSIS THALIANA XYLOGEN PROTEIN 2 (XYP2) but not XYP1 is specifically expressed in vascular tissues. The double loss-of-function mutant of xyp1/2 showed discontinuous TE formation, indicating that communication between TE is important for the correct formation of TE cell files [61,62].

XYLEM CYSTEINE PROTEASE1 (XCP1) and XCP2 contribute to micro-autolysis within the tonoplast, as well as the mega-autolysis after the tonoplast ruptures [63]. The S1-type nuclease ZINNIA ENDONUCLEASE 1 (ZEN1) was shown to be necessary for post-mortem DNA degradation during TE differentiation in Z. elegans cell cultures [64]. In Arabidopsis, BIFUNCTIONAL NUCLEASE1 (BFN1), which shows a high sequence similarity to ZEN1, is expressed in differentiating xylem and exhibits DNase activity, suggesting that it may have a similar function [65,66]. In Arabidopsis, METACASPASE9 (MC9) is required for the clearance of cellular contents after cell death in xylem vessel elements, which is likely mediated by papain-like cysteine proteases $[67,68]$ (Figure 2b).

\section{Conclusion}

Improvements in imaging and tissue culture techniques have led to an improved understanding of both SE and TE differentiation. For example, serial block-face scanning electron microscopy has recently been used to study the morphological changes during SE differentiation in greater detail [18,34]. Many similarities have between the two process have been revealed. NAC TFs serve as key regulators of both SE and TE differentiation, with the couplets NAC45/86 and VND6/7 respectively controlling the two processes $[18,56,58]$. Both differentiation programs appear to require active BES1 signalling and are inhibited by CLE peptides [40,42,51,52]. In addition, polarly-localized signalling components on the plasma membrane, including BRX, OPS and xylogen, are necessary to ensure the correct pace and continuity of differentiation in both types of conduit [39,41,62]. Despite these similarities, the differentiation processes are executed by different functional components in these conductive cells. Furthermore, the regulatory networks known to be involved in SE differentiation are relatively incomplete, whereas those controlling TE differentiation are better understood. This difference may be due to the early discovery of the key regulators $V N D 6 / 7$, which allowed experimental initiation of TE differentiation from other cell types, greatly advancing research in this field [56]. Similar regulators of SE differentiation, NAC45/86, have only been recently discovered, and their capacity to induce cytosol degradation is not yet fully understood [18]. Nonetheless, ectopic overexpression of NAC45/86 in an inducible manner could offer improved avenues for the study of 
the cellular changes during SE differentiation, particularly with regard to the temporal order of events. Further advances may also become possible by exploiting the ability of the VISUAL tissue culture system to induce ectopic SE-like differentiation [36]. However, a full understanding of SE differentiation and function can only be achieved by incorporating the insights gleaned from these approaches into in planta experiments. 


\section{Figure captions}

Figure 1.

Overview of the vascular tissue differentiation. (a) Phloem sieve elements undergo a cellular rearrangement in which organelles, such as plastids, mitochondria, endoplasmic reticulum are modified and reoriented toward the parietal walls of the cell. The nucleus changes its shape and eventually becomes degraded. P-proteins are formed in early stage and retained after nuclear degradation. In mature SEs, P-protein filaments are found at the side walls of the cell. At maturity, SE forms sieve plates - highly perforated pores between SEs. SEs are associated with companion cells (CCs) through branched plasmodesmata, also known as phloem pore units. CCs are characterized by their dense cytoplasm as they contain increased number of organelles, such as plastids and mitochondria. (b) Xylem tracheary elements undergo programmed cell death. After vacuole ruptures, organelles including the nucleus are degraded. The deposition of secondary cell walls occurs before and after the vacuole rupture. Lignins are deposited in a specific pattern at lateral walls of TEs. The end walls of a vessel element become modified to form perforation plates. However, the timing of when it is formed is unclear.

Figure 2.

Regulatory networks in SE and TE differentiation. (a) OPS regulates SE differentiation by suppressing activity of BIN2, a putative suppressor of BES1. BRX restricts the expression of BAM3, the receptor of CLE45 signalling which inhibits SE differentiation. The interplay of BRX and OPS is not fully understood yet. In addition, the $\mathrm{PIP}_{2}$ level regulated by CVP2 has a major impact on SE differentiation. The MYB type TF APL regulates the expression of NAC45/86. NENs as downstream targets of the APL-NACs pathway play a key role in SE enucleation. APL expression is downregulated by NAC20. (b) Thermospermine levels controlled by ACL5 regulate the expression of the TE differentiation suppressor SAC51. At the same time, the key regulators of TE differentiation, VND6/7, also directly control expression of SAC51. VND6/7 control the expression of MYB46/83, key regulators of secondary cell wall thickening, and MC9 that is required for autolytic degradation of cellular contents. The expression of XCP1/2 proteases are also controlled by VND6/7 and participate in both vacuolar rupture and subsequent autolysis. VNI2 interacts with VND7, thus repressing its function. ZEN1/BFN1 are thought to facilitate the breakdown of the nucleus. Xylogen is a positive signal for TE differentiation, while CLE41/44 signaling has an inhibitory effect. Uncertain interactions are represented with dotted lines. 


\section{Acknowledgements}

The authors thank Bonghee Heo for kind help with graphical illustrations. We also thank S. el-Showk for critically reading and proof-reading the manuscript. The Y.H. laboratory is funded by the Academy of Finland Centre of Excellence programme, the Gatsby Foundation, the Biotechnology and Biological Sciences Research Council, the University of Helsinki, the European Research Council Advanced Investigator Grant Symdev (No. 323052) and Tekes (the Finnish Funding Agency for Technology and Innovation). 


\section{References}

1. Notaguchi M, Okamoto S: Dynamics of Long-distance Signaling via Plant Vascular Tissues. Front Plant Sci 2015, 6.

2. •- Lacombe B, Achard P: Long-distance transport of phytohormones through the plant vascular system. Curr Opin Plant Biol 2016, 34:1-8.

This review summerizes current knowledge about phytohormones that are transported long-distance through vascular tissues.

3. Turnbull CG, Lopez-Cobollo RM: Heavy traffic in the fast lane: long-distance signalling by macromolecules. New Phytol 2013, 198:33-51.

4. Kehr J, Buhtz A: Long distance transport and movement of RNA through the phloem. $J$ Exp Bot 2008, 59:85-92.

5. Lucas WJ, Groover A, Lichtenberger R, Furuta K, Yadav SR, Helariutta Y, He XQ, Fukuda H, Kang J, Brady SM, et al.: The plant vascular system: evolution, development and functions. J Integr Plant Biol 2013, 55:294-388.

6. - Knoblauch M, Knoblauch J, Mullendore DL, Savage JA, Babst BA, Beecher SD, Dodgen AC, Jensen KH, Holbrook NM: Testing the Munch hypothesis of long distance phloem transport in plants. Elife 2016, 5.

Authors in this study provide experimental evidences that support pressure flow theory proposed by Munch in 1930.

7. Kim HK, Park J, Hwang I: Investigating water transport through the xylem network in vascular plants. J Exp Bot 2014, 65:1895-1904.

8. •• De Rybel B, Mähönen AP, Helariutta Y, Weijers D: Plant vascular development: from early specification to differentiation. Nat Rev Mol Cell Biol 2016, 17:30-40.

This review describes current understanding of early vascular pattern formation and late differentiation of vascular tissues. Recent knowledge about molecular regulation is also covered.

9. Bishopp A, Help H, El-Showk S, Weijers D, Scheres B, Friml J, Benkova E, Mahonen AP, Helariutta Y: A mutually inhibitory interaction between auxin and cytokinin specifies vascular pattern in roots. Curr Biol 2011, 21:917-926.

10. Bishopp A, Lehesranta S, Vaten A, Help H, El-Showk S, Scheres B, Helariutta K, Mahonen AP, Sakakibara H, Helariutta Y: Phloem-transported cytokinin regulates polar auxin transport and maintains vascular pattern in the root meristem. Curr Biol 2011, 21:927932.

11. De Rybel B, Adibi M, Breda AS, Wendrich JR, Smit ME, Novak O, Yamaguchi N, Yoshida S, Van Isterdael G, Palovaara J, et al.: Plant development. Integration of growth and patterning during vascular tissue formation in Arabidopsis. Science 2014, 345:1255215.

12. Furuta KM, Hellmann E, Helariutta Y: Molecular control of cell specification and cell differentiation during procambial development. Annu Rev Plant Biol 2014, 65:607-638.

13. Scarpella E, Meijer AH: Pattern formation in the vascular system of monocot and dicot plant species. New Phytol 2004, 164:209-242.

14. Evert RF: Phloem: Cell Types and Developmental Aspects. In Esau's Plant Anatomy. Edited by: John Wiley \& Sons, Inc.; 2006:357-405. 
15. Behnke HD: [Sieve-tube plastids of monocotyledons : Comparative investigations of the fine structure and distribution of specific plastids]. Planta 1968, 84:174-184.

16. Esser K, Kubitzki K, Runge M, Schnepf E, Ziegler H: Progress in Botany / Fortschritte der Botanik Morphology • Physiology • Genetics • Taxonomy • Geobotany / Morphologie • Physiologie · Genetik · Systematik · Geobotanik. 1983.

17. Evert RF, Eschrich W, Eichhorn SE: P-protein distribution in mature sieve elements of Cucurbita maxima. Planta 1972, 109:193-210.

18. •• Furuta KM, Yadav SR, Lehesranta S, Belevich I, Miyashima S, Heo J-o, Vatén A, Lindgren O, Rybel BD, Isterdael GV, et al.: Arabidopsis NAC45/86 direct sieve element morphogenesis culminating in enucleation. Science 2014, 345:933-937.

The authors of this study show how NAC transcription factors control the enucleation of sieve elements via exonucleases.

19. Lalonde S, Franceschi VR, Frommer WB: Companion Cells. In eLS. Edited by: John Wiley \& Sons, Ltd; 2001.

20. Fukuda H: Programmed cell death during vascular system formation. Cell Death Differ 1997, 4:684-688.

21. Fukuda H: Tracheary Element Differentiation. Plant Cell 1997, 9:1147-1156.

22. Bollhoner B, Prestele J, Tuominen H: Xylem cell death: emerging understanding of regulation and function. $J$ Exp Bot 2012, 63:1081-1094.

23. Behnke HD, Sjolund RD: Sieve Elements Comparative Structure, Induction and Development. 1990.

24. Oparka KJ, Turgeon R: Sieve elements and companion cells-traffic control centers of the phloem. Plant Cell 1999, 11:739-750.

25. Oda Y, Fukuda H: Secondary cell wall patterning during xylem differentiation. Curr Opin Plant Biol 2012, 15:38-44.

26. Evert RF: Xylem: Cell Types and Developmental Aspects. In Esau's Plant Anatomy. Edited by: John Wiley \& Sons, Inc.; 2006:255-290.

27. •• Escamez S, Tuominen H: Programmes of cell death and autolysis in tracheary elements: when a suicidal cell arranges its own corpse removal. J Exp Bot 2014, 65:1313-1321.

This review provides detailed description about xylem differentiation. Molecular mechanisms and dynamic cellular changes during xylem differentiation are well described.

28. Schuetz M, Benske A, Smith RA, Watanabe Y, Tobimatsu Y, Ralph J, Demura T, Ellis B, Samuels AL: Laccases direct lignification in the discrete secondary cell wall domains of protoxylem. Plant Physiol 2014, 166:798-807.

29. Fukuda H: Signaling, transcriptional regulation, and asynchronous pattern formation governing plant xylem development. Proc Jpn Acad Ser B Phys Biol Sci 2016, 92:98-107.

30. Oda Y, Fukuda H: Initiation of cell wall pattern by a Rho- and microtubule-driven symmetry breaking. Science 2012, 337:1333-1336.

31. • Zhong R, Ye ZH: Secondary cell walls: biosynthesis, patterned deposition and transcriptional regulation. Plant Cell Physiol 2015, 56:195-214.

Comprehensive knowledge on secondary cell wall deposition and biosynthesis is introduced in this review. This review also includes information about transcriptional regulation of secondary cell wall formation.

32. Bonke M, Thitamadee S, Mähönen AP, Hauser M-T, Helariutta Y: APL regulates vascular tissue identity in Arabidopsis. Nature 2003, 426:181-186. 
33. Truernit E, Bauby H, Dubreucq B, Grandjean O, Runions J, Barthélémy J, Palauqui J-C: Highresolution whole-mount imaging of three-dimensional tissue organization and gene expression enables the study of Phloem development and structure in Arabidopsis. Plant Cell 2008, 20:1494-1503.

34. • Dettmer J, Ursache R, Campilho A, Miyashima S, Belevich I, O'Regan S, Mullendore DL, Yadav SR, Lanz C, Beverina L, et al.: CHOLINE TRANSPORTER-LIKE1 is required for sieve plate development to mediate long-distance cell-to-cell communication. $\mathrm{Nat}$ Commun 2014, 5:4276.

This article describes the importance of choline for the correct transformation of plasmodesmata to sieve plate pores, one of the few known regulations of specific morphological changes in SE differentiation.

35. Xie B, Wang X, Zhu M, Zhang Z, Hong Z: CalS7 encodes a callose synthase responsible for callose deposition in the phloem. Plant $J$ 2011, 65:1-14.

36. • Kondo Y, Nurani AM, Saito C, Ichihashi Y, Saito M, Yamazaki K, Mitsuda N, Ohme-Takagi M, Fukuda H: Vascular Cell Induction Culture System Using Arabidopsis Leaves (VISUAL) Reveals the Sequential Differentiation of Sieve Element-like Cells. Plant Cell 2016.

The culture system described in this article allows the study not only of xylem but also phloem differentiation. Although not all morphological changes of SE differentiation occur, this likely will help to identify novel regulatory elements.

37. Kang YH, Hardtke CS: Arabidopsis MAKR5 is a positive effector of BAM3-dependent CLE45 signaling. EMBO rep 2016, 17:1145-1154.

38. Scacchi E, Osmont KS, Beuchat J, Salinas P, Navarrete-Gómez M, Trigueros M, Ferrándiz C, Hardtke CS: Dynamic, auxin-responsive plasma membrane-to-nucleus movement of Arabidopsis BRX. Development 2009, 136:2059-2067.

39. Truernit E, Bauby H, Belcram K, Barthélémy J, Palauqui J-C: OCTOPUS, a polarly localised membrane-associated protein, regulates phloem differentiation entry in Arabidopsis thaliana. Development 2012, 139:1306-1315.

40. Anne P, Azzopardi M, Gissot L, Beaubiat S, Hématy K, Palauqui J-C: OCTOPUS Negatively Regulates BIN2 to Control Phloem Differentiation in Arabidopsis thaliana. Curr Biol 2015, 25:2584-2590.

41. Scacchi E, Salinas P, Gujas B, Santuari L, Krogan N, Ragni L, Berleth T, Hardtke CS: Spatiotemporal sequence of cross-regulatory events in root meristem growth. Proc Natl Acad Sci U S A 2010, 107:22734-22739.

42. Depuydt S, Rodriguez-Villalon A, Santuari L, Wyser-Rmili C, Ragni L, Hardtke CS: Suppression of Arabidopsis protophloem differentiation and root meristem growth by CLE45 requires the receptor-like kinase BAM3. Proc Natl Acad Sci U S A 2013, 110:7074-7079.

43. •• Rodriguez-Villalon A, Gujas B, Kang YH, Breda AS, Cattaneo P, Depuydt S, Hardtke CS: Molecular genetic framework for protophloem formation. Proc Natl Acad Sci U S A 2014, 111:11551-11556.

This study sheds light on the regulatory interactions in early SE differentiation inclduing BRX, OPS and CLE45/BAM3.

44. Rodriguez-Villalon A, Gujas B, van Wijk R, Munnik T, Hardtke CS: Primary root protophloem differentiation requires balanced phosphatidylinositol-4,5-biphosphate levels and systemically affects root branching. Development 2015, 142:1437-1446. 
45. Carlsbecker A, Lee J-Y, Roberts CJ, Dettmer J, Lehesranta S, Zhou J, Lindgren O, MorenoRisueno MA, Vatén A, Thitamadee $S$, et al.: Cell signalling by microRNA165/6 directs gene dose-dependent root cell fate. Nature 2010, 465:316-321.

46. Donner TJ, Sherr I, Scarpella E: Regulation of preprocambial cell state acquisition by auxin signaling in Arabidopsis leaves. Development 2009, 136:3235-3246.

47. Mähönen AP, Bishopp A, Higuchi M, Nieminen KM, Kinoshita K, Törmäkangas K, Ikeda Y, Oka A, Kakimoto T, Helariutta Y: Cytokinin signaling and its inhibitor AHP6 regulate cell fate during vascular development. Science 2006, 311:94-98.

48. Miyashima S, Koi S, Hashimoto T, Nakajima K: Non-cell-autonomous microRNA165 acts in a dose-dependent manner to regulate multiple differentiation status in the Arabidopsis root. Development 2011, 138:2303-2313.

49. Müller CJ, Valdés AE, Wang G, Ramachandran P, Beste L, Uddenberg D, Carlsbecker A: PHABULOSA Mediates an Auxin Signaling Loop to Regulate Vascular Patterning in Arabidopsis. Plant Physiol 2016, 170:956-970.

50. Ursache R, Miyashima S, Chen Q, Vatén A, Nakajima K, Carlsbecker A, Zhao Y, Helariutta Y, Dettmer J: Tryptophan-dependent auxin biosynthesis is required for HD-ZIP IIImediated xylem patterning. Development 2014, 141:1250-1259.

51. Hirakawa Y, Shinohara H, Kondo Y, Inoue A, Nakanomyo I, Ogawa M, Sawa S, Ohashi-Ito K, Matsubayashi Y, Fukuda H: Non-cell-autonomous control of vascular stem cell fate by a CLE peptide/receptor system. Proc Natl Acad Sci U S A 2008, 105:15208-15213.

52. • Kondo Y, Ito T, Nakagami H, Hirakawa Y, Saito M, Tamaki T, Shirasu K, Fukuda H: Plant GSK3 proteins regulate xylem cell differentiation downstream of TDIF-TDR signalling. Nat Commun 2014, 5:3504.

This article explains the downstream signalling of TDIF/TDR and the overlap with brassinosteroid signalling.

53. Kondo Y, Fujita T, Sugiyama M, Fukuda H: A Novel System for Xylem Cell Differentiation in Arabidopsis thaliana. Mol Plant 2015, 8:612-621.

54. Pyo H, Demura T, Fukuda H: TERE; a novel cis-element responsible for a coordinated expression of genes related to programmed cell death and secondary wall formation during differentiation of tracheary elements. Plant $J$ 2007, 51:955-965.

55. Zhong R, Lee C, Ye Z-H: Global Analysis of Direct Targets of Secondary Wall NAC Master Switches in Arabidopsis. Mol Plant 2010, 3:1087-1103.

56. Ohashi-Ito K, Oda Y, Fukuda H: Arabidopsis VASCULAR-RELATED NAC-DOMAIN6 directly regulates the genes that govern programmed cell death and secondary wall formation during xylem differentiation. Plant Cell 2010, 22:3461-3473.

57. Zhong R, Ye Z-H: MYB46 and MYB83 bind to the SMRE sites and directly activate a suite of transcription factors and secondary wall biosynthetic genes. Plant Cell Physiol 2012, 53:368-380.

58. Yamaguchi M, Mitsuda N, Ohtani M, Ohme-Takagi M, Kato K, Demura T: VASCULARRELATED NAC-DOMAIN7 directly regulates the expression of a broad range of genes for xylem vessel formation. Plant $J$ 2011, 66:579-590.

59. Muñiz L, Minguet EG, Singh SK, Pesquet E, Vera-Sirera F, Moreau-Courtois CL, Carbonell J, Blázquez MA, Tuominen H: ACAULIS5 controls Arabidopsis xylem specification through the prevention of premature cell death. Development 2008, 135:2573-2582.

60. Imai A, Hanzawa Y, Komura M, Yamamoto KT, Komeda Y, Takahashi T: The dwarf phenotype of the Arabidopsis acl5 mutant is suppressed by a mutation in an upstream ORF of a bHLH gene. Development 2006, 133:3575-3585. 
61. Kobayashi Y, Motose H, Iwamoto K, Fukuda H: Expression and Genome-Wide Analysis of the Xylogen-Type Gene Family. Plant Cell Physiol 2011, 52:1095-1106.

62. Motose $\mathrm{H}$, Sugiyama M, Fukuda $\mathrm{H}$ : A proteoglycan mediates inductive interaction during plant vascular development. Nature 2004, 429:873-878.

63. Avci U, Petzold HE, Ismail IO, Beers EP, Haigler CH: Cysteine proteases XCP1 and XCP2 aid micro-autolysis within the intact central vacuole during xylogenesis in Arabidopsis roots. Plant $J$ 2008, 56:303-315.

64. Ito J, Fukuda H: ZEN1 Is a Key Enzyme in the Degradation of Nuclear DNA during Programmed Cell Death of Tracheary Elements. Plant Cell 2002, 14:3201-3211.

65. Farage-Barhom S, Burd S, Sonego L, Perl-Treves R, Lers A: Expression analysis of the BFN1 nuclease gene promoter during senescence, abscission, and programmed cell deathrelated processes. $J$ Exp Bot 2008, 59:3247-3258.

66. Perez-Amador MA, Abler ML, De Rocher EJ, Thompson DM, van Hoof A, LeBrasseur ND, Lers A, Green PJ: Identification of BFN1, a bifunctional nuclease induced during leaf and stem senescence in Arabidopsis. Plant Physiol 2000, 122:169-180.

67. Bollhöner B, Zhang B, Stael S, Denancé N, Overmyer K, Goffner D, Van Breusegem F, Tuominen H: Post mortem function of AtMC9 in xylem vessel elements. New Phytol 2013, 200:498-510.

68. Escamez S, André D, Zhang B, Bollhöner B, Pesquet E, Tuominen H: METACASPASE9 modulates autophagy to confine cell death to the target cells during Arabidopsis vascular xylem differentiation. Biology Open 2016, 5:122-129. 